\title{
Statistical analysis and significance testing of serial analysis of gene expression data using a Poisson mixture model Scott D Zuyderduyn*1,2
}

\author{
Address: ${ }^{1}$ Victor Ling Laboratory, Department of Cancer Genetics and Developmental Biology, BC Cancer Research Centre, 675 West 10 th Ave., \\ Vancouver, Canada and ${ }^{2}$ Graduate Program, Department of Biochemistry and Molecular Biology, Faculty of Medicine, University of British \\ Columbia, 2350 Health Sciences Mall, Vancouver, Canada \\ Email: Scott D Zuyderduyn* - scottz@bccrc.ca \\ * Corresponding author
}

Published: 2 August 2007

BMC Bioinformatics 2007, 8:282 doi:10.1/86/147|-2/05-8-282
Received: 10 May 2007

Accepted: 2 August 2007

This article is available from: http://www.biomedcentral.com//47/-2105/8/282

C) 2007 Zuyderduyn; licensee BioMed Central Ltd.

This is an Open Access article distributed under the terms of the Creative Commons Attribution License (http://creativecommons.org/licenses/by/2.0), which permits unrestricted use, distribution, and reproduction in any medium, provided the original work is properly cited.

\begin{abstract}
Background: Serial analysis of gene expression (SAGE) is used to obtain quantitative snapshots of the transcriptome. These profiles are count-based and are assumed to follow a Binomial or Poisson distribution. However, tag counts observed across multiple libraries (for example, one or more groups of biological replicates) have additional variance that cannot be accommodated by this assumption alone. Several models have been proposed to account for this effect, all of which utilize a continuous prior distribution to explain the excess variance. Here, a Poisson mixture model, which assumes excess variability arises from sampling a mixture of distinct components, is proposed and the merits of this model are discussed and evaluated.
\end{abstract}

Results: The goodness of fit of the Poisson mixture model on 15 sets of biological SAGE replicates is compared to the previously proposed hierarchical gamma-Poisson (negative binomial) model, and a substantial improvement is seen. In further support of the mixture model, there is observed: I) an increase in the number of mixture components needed to fit the expression of tags representing more than one transcript; and 2) a tendency for components to cluster libraries into the same groups. A confidence score is presented that can identify tags that are differentially expressed between groups of SAGE libraries. Several examples where this test outperforms those previously proposed are highlighted.

Conclusion: The Poisson mixture model performs well as a) a method to represent SAGE data from biological replicates, and b) a basis to assign significance when testing for differential expression between multiple groups of replicates. Code for the R statistical software package is included to assist investigators in applying this model to their own data.

\section{Background}

Serial analysis of gene expression (SAGE) is a technique for obtaining a quantitative, global snapshot of the transcriptome [1]. The method extracts short sequence tags (containing 10, 17, or 22 bp of information, depending on the protocol) from each messenger RNA; these are seri- ally ligated, cloned and sequenced, and can then be counted to obtain a profile [1-3]. SAGE has been used to study the transcriptome of a variety of tissue and cell types from a diverse set of organisms. The technique was originally conceived to study the cancer transcriptome, and has been utilized extensively to do so. 
As a counting technology, SAGE produces profiles consisting of a digital output that is quantitative in nature. For example, a statement can be made with reasonable certainty that a SAGE tag observed 30 times in a library of 100,000 tags corresponds to a transcript that comprises $0.03 \%$ of the total transcriptome; the same statement cannot be made reliably with analog values, like that obtained from a microarray. Accordingly, a reliable statistical model should account for the discrete, count-based nature of SAGE observations. When testing for differential expression between groups, where each group can contain multiple libraries, statistical methods that incorporate a continuous probability distribution (e.g. the Normal distribution assumed by Student's $t$-test) should be avoided. Indeed, such tests require tag counts be normalized by division with the total library size; this removal of library size from the set of sufficient statistics discards an informative facet of the data.

The sampling of SAGE tags can be modeled by the Binomial distribution which describes the probability of observing a number of successes in a series of Bernoulli trials. Here, the library size corresponds to the number of trials and the count of a particular tag is the number of successful trial outcomes. When the probability of an event is small, the Binomal distribution approaches the Poisson distribution as the number of trials increases. This is the case for SAGE (since the tag counts are small relative to a large library size), so the form of the Poisson and Binomial distribution is essentially the same. A fortunate characteristic of both of these distributions is that they are a function of a single parameter only, since the variance in observed data is directly calculable from the mean.

However, in practice, the variance of SAGE data is often larger than can be explained by sampling alone. Several authors have attributed this effect, termed "overdispersion", to a latent biological variability [4-6]. [4] refers to this as "between"-library variability, as opposed to "within"-library variability caused by sampling. Examples of factors that could contribute to this variability are numerous, including: sample preparation or quality, artefacts intrinsic to the library construction protocol, differences in gene transcription due to environment, or the intrinsic stability or regulatory complexity of transcription at a particular locus. This will adversely affect statistical analysis because additional variance results in an overstated significance. Procedures for using hierarchical models which incorporate a continuous prior distribution to explain the excess variance have been presented for both the Binomial (viz. beta-binomial using logistic regression [5], $\mathrm{t}_{\mathrm{w}}$-test [4], or Bayes error rate [7]) and Poisson (viz. negative binomial a.k.a. hierarchical gamma-Poisson using log-linear regression [6]) distributions. Attempts to use the log-normal and inverse-Gaussian as prior distribu- tions (both of these have longer tails) did not show an appreciable improvement and are computationally difficult to fit (data not shown).

Here, it is argued that the excess variation is due to a mixing of two or more distinct Poisson (or Binomial) components, and this mixing is the predominant source of total variation. This assumption corresponds to a finite mixture model, which have found wide applicability in several fields (for a general introduction, McLachlan and Peel is a good source [8]). To illustrate, consider a tag from ten SAGE libraries of equal size (e.g. 100,000 tags) that has observed counts where half are realizations of an expression of 0.0003 and the other half of 0.0004 . As a result, the probability distribution of observing a particular tag count will be a combination of these two components (Figure 1). Note the similarity between the shapes of the probability distributions estimated from a fitted negative binomial (which assumes sampling variability drawn from a latent biological variability) and a Poisson mixture model (which assumes a set of independent components, each having sampling variability only).

If the Poisson mixture model is an accurate foundation to explain SAGE observations, it is attractive for several reasons. First, this approach does not rely on a vague and continuous prior distribution to explain additional variance. Rather, the model asserts that a gene's expression level will take on one of a number of distinct states. Second, overdispersed models applied to SAGE data tend to show a wide range of excess variation; in many cases, far greater than can be attributed to counting. This is a troubling prospect for studies that utilize a limited number of libraries (e.g. pair-wise comparisons), since the observed count may differ wildly from the underlying expression. If a mixture model provides an improved fit to SAGE data, this concern would be assuaged. Finally, mixture models, by nature, allow for the concept of subsets (or latent classes) in the expression values of each tag. Dysregulation of genes in disease processes such as cancer are often observed in only a proportion of profiled samples, and these will be naturally identified during model fitting. This property can also be utilized to identify sets of coexpressed genes.

\section{Results \\ Goodness of fit}

In order to evaluate the efficacy of a mixture model approach, a comparison of the goodness of fit of this and previously described models on 15 sets of biological replicates from publicly available SAGE data was performed (see Methods).

Goodness of fit was assessed for: 1) the canonical log-linear (Poisson) model, 2) negative binomial (i.e. hierarchi- 


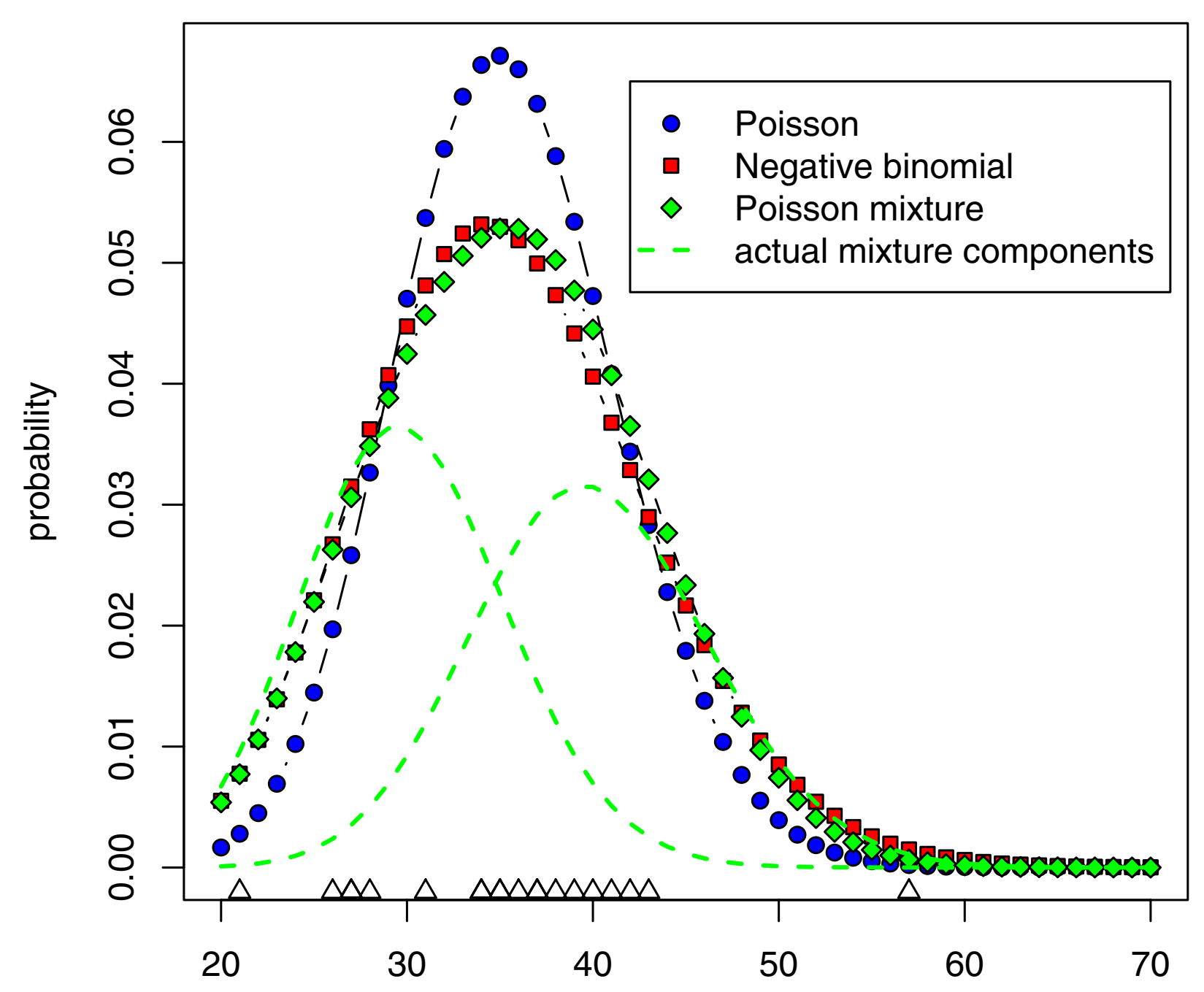

tag count

\section{Figure I}

Probability density of several models applied to data generated from two Poisson components. 10 observations were randomly drawn from each of two Poisson distributions, one with a mean of 30 , the other 40 . The values drawn from the first component were $(40,34,37,28,31,21,41,27,34,27)$ and the values drawn from the second component were $(36,42$, $26,57,43,37,38,39,35,35$ ). The probability densities are shown for a single Poisson distribution, the negative binomial distribution, and a two-component Poisson mixture distribution using maximum likelihood estimates (see Methods). The probability densities of the individual Poisson components from which the data were actually drawn are also shown. The individual observations are represented by triangles at the bottom of the plot. 
cal gamma-Poisson or overdispersed log-linear) model, and 3) k-component Poisson mixture model (see Methods for a brief description of each). Since maximum likelihood estimation (MLE) is used to fit each of these models, the log-likelihood was the basis for assessing relative goodness of fit. A comparison of the Akaike information criterion (AIC) [9] and Bayesian information criterion (BIC) [10] (both of which use the log-likelihood and a term to penalize a model for estimating a larger number of parameters) was performed on each of the datasets (Table 1).

As expected, the canonical Poisson model, which does not account for excess variance, performs poorly in all cases. The Poisson mixture model consistently outperforms the negative binomial model regardless of the metric used. The competitiveness of the negative binomial model is perhaps not surprising since a comparison of the fit of these two models to simulated data indicates that the negative binomial can often fit better to data generated from a two-component Poisson mixture. This becomes more problematic as the component means draw closer (data not shown, Figure 1 is a good example). However, several hypotheses can be tested to further strengthen the case for the mixture model approach. These are considered in turn.

\section{Tags with ambiguous mappings are represented by a greater number of components}

Consider an idealized situation where a gene's expression can take on one of two states (and can therefore be modelled by a two-component Poisson mixture). A significant proportion of SAGE tags are ambiguous (correspond to more than one gene) and, under the idealized model, would result in tag counts that are modelled by $2 \mathrm{~g}$ components (where $\mathrm{g}$ is the number of expressed genes the tag corresponds to). Therefore, the number of components in the mixture should be higher for ambiguous tags.

Simply partitioning the data into ambiguous and unambiguous tags and comparing the number of components is unlikely to be informative since, for any given ambiguous tag, it is not known how many of the possible genes are actually expressed. However, two normal brain libraries used in this study were generated using LongSAGE (GSM31931 and GSM31935), which provides 17 base pairs of information rather than 10 . The tag sequences in these libraries were shortened before inclusion in the normal brain dataset used in the previous section. However, by comparing the shortened tag list to the original library, tags that actually correspond to two or more LongSAGE tag sequences (and presumably represent different transcripts) were identified. Tags counts of one or two were considered artefacts of PCR amplification or sequencing and were not used in this determination.
The number of ambiguous and unambiguous tags was tallied for each estimated number of components (Table 2). Ambiguous tags are represented more highly in the set of model fits that consist of a larger number of components. This effect, which is statistically significant, is consistent with the mixture model hypothesis.

\section{Component assignment of libraries is non-random}

If the mixture model approach holds, then the Poisson components should cluster the libraries into recurring groups. Such an enrichment of certain component assignments would be expected for a number of reasons. Two possibilities are: a) if one or more libraries are mislabelled, the tag expression in those libraries should show a preferential assignment to a separate component; and b) if the genes corresponding to a set of tags are co-expressed, the component assignment should be similar amongst these genes. Conversely, if the negative binomial model is more appropriate then component assignments should essentially be random, since the distribution assumed to give rise to biological variability is continuous and unconditional.

For each of the datasets, the component assignments for tags where the estimated number of components is two were tallied. The individual assignment was based on the component with the highest posterior probability, given a tag count and library size. In all cases, there were a significant number of tags where the parent libraries were partitioned into the same two components (Table 3). For example, in the AML libraries containing the cytogenetic abnormality $\mathrm{t}(8 ; 21)$, of the 225 tags that had expression that could be fit to two Poisson components, 110 were partitioned in the form -++-- ( $p=4.5 \mathrm{E}-67$; Binomial test). In other words, almost half of the tags that fit to two components were assigned to a single component configuration (for 5 libraries, $\left(2^{5} / 2\right)-1=15$ such configurations are possible).

\section{Determining differentially expressed genes}

In previously described overdispersed models, the identity of a library is included a priori as a model covariate. Significance is then determined by testing the null hypothesis that the fitted $\beta$ coefficient for this covariate is zero $[5,6]$. A Bayesian significance score has also described, although this was developed using a beta-binomial model [7]. In contrast, the Poisson mixture model does not require the identity of the libraries be included (although the addition of such covariates is possible). Rather, once a mixture model has been fit, the posterior probabilities of membership in a particular component given the observed tag counts can be used to determine how well the components can differentiate between two or more sample types (e.g. normal versus cancer). Here, a score is presented based on the confidence that a sample is of type 
Table I: Comparison of model fits to a single group of biological replicates

\begin{tabular}{|c|c|c|c|c|c|c|c|c|c|}
\hline & \multirow[b]{2}{*}{$\mathbf{N}$} & \multirow[b]{2}{*}{ tags } & \multirow[b]{2}{*}{$\mathbf{k}$} & \multicolumn{3}{|c|}{ mean AIC } & \multicolumn{3}{|c|}{ mean BIC } \\
\hline & & & & Poisson & Negbin & Mixture & Poisson & Negbin & Mixture \\
\hline \multicolumn{10}{|l|}{ BRAIN } \\
\hline astrocytoma & 14 & $|14|$ & 2.6 & 238.2 & 105.2 & 103.6 & 238.9 & 106.5 & 106.3 \\
\hline ependymoma & 10 & 1205 & 2.3 & 152.4 & 80.9 & 75.0 & 152.7 & 81.5 & 76.1 \\
\hline glioblastoma & 7 & 1197 & 2.3 & 139.5 & 57.6 & 53.0 & 139.4 & 57.5 & 52.8 \\
\hline medulloblastoma & 18 & 1045 & 2.7 & 280.6 & 128.7 & 128.7 & 281.5 & 130.5 & 132.6 \\
\hline normal & 8 & 1099 & 2.4 & 156.8 & 68.0 & 59.8 & 156.8 & 68.2 & 60.1 \\
\hline \multicolumn{10}{|l|}{ AML } \\
\hline $\operatorname{inv}(16)$ & 5 & 900 & 1.7 & 68.9 & 39.3 & 37.6 & 68.5 & 38.5 & 36.7 \\
\hline$t(8 ; 21)$ & 5 & 1037 & 1.3 & 52.3 & 34.1 & 33.5 & 51.9 & 33.3 & 32.9 \\
\hline $\mathrm{t}(15 ; 17)$ & 5 & 709 & 1.8 & 127.7 & 46.0 & 38.9 & 127.3 & 45.2 & 37.9 \\
\hline $\mathrm{t}(9 ; 1 \mathrm{I})$ de novo & 4 & 954 & 1.8 & 58.5 & 34.6 & 30.1 & 57.9 & 33.4 & 28.5 \\
\hline$t(9 ; 11)$ treatment & 3 & 1061 & 1.5 & 42.9 & 32.0 & 20.9 & 42.0 & 30.2 & 19.1 \\
\hline \multicolumn{10}{|l|}{ BREAST } \\
\hline normal & 6 & 1259 & 1.8 & 71.6 & 43.9 & 41.6 & 71.4 & 43.5 & 41.0 \\
\hline DCIS & 4 & 598 & 1.3 & 25.5 & 24.0 & 21.0 & 24.9 & 22.8 & 20.1 \\
\hline invasive & 3 & 1069 & 2.0 & 60.4 & 27.8 & 22.8 & 59.5 & 26.0 & 20.1 \\
\hline \multicolumn{10}{|l|}{ SKIN } \\
\hline normal & 4 & 1015 & 1.6 & 33.8 & 24.6 & 22.2 & 33.2 & 23.4 & 20.9 \\
\hline melanoma & 3 & 992 & 1.8 & 38.2 & 24.0 & 19.6 & 37.3 & 22.2 & 17.4 \\
\hline
\end{tabular}

SAGE tag counts from fifteen sets of biological replicates were fit to log-linear (Poisson), negative binomial (overdispersed log-linear), and Poisson mixture models. The table contains the number of replicates $(\mathrm{N})$, tags tested, and mean number of mixture components $(\mathrm{k})$. For each model, mean goodness of fit scores calculated using Akaike's Information Criterion (AIC) and Bayesian Information Criterion (BIC) are shown. For both scores, a lower value indicates a better fit.

$\omega$ given that it arises from component(s) k. Using Bayes Theorem, one can derive the following expression [see Additional file 1]

$$
P(\omega \mid k)=\sum_{j}^{\omega} \tau_{j k} / \sum_{j} \tau_{j k}
$$

where $\omega$ is the set of libraries corresponding to some label of interest (e.g. normal or cancer) and $\tau_{\mathrm{jk}}$ is the posterior probability of the tag count from library $\mathrm{j}$ arising from component(s) $\mathrm{k}$. Using this expression, one can deter- mine which tags have a set of mixture components that are closely linked with the sample type(s) of interest.

To illustrate, SAGE libraries from normal brain $(\mathrm{n}=8)$ and ependymoma $(n=10)$ (a type of brain tumour) were analyzed using both the overdispersed log-linear and Poisson mixture models. In the former case, significance was calculated using the method described in [5] (see also example $\mathrm{R}$ code in Methods). In the latter case, the method described above was used. A plot of the two sets of scores shows a moderate correlation and tags that are

Table 2: Mean number of mixture model components

\begin{tabular}{lcccc}
\hline Library & $\boldsymbol{k}$ & Unambiguous & Ambiguous & Significance \\
\hline GSM31931 & 1 & 93 & 0 & $p<2.2 \mathrm{E}-16\left(\chi^{2}=134.1 ; \mathrm{df}=4\right)$ \\
& 2 & 405 & 15 & \\
& 3 & 210 & 32 & \\
GSM31935 & 4 & 27 & 12 & $p=1.8 \mathrm{E}-6\left(\chi^{2}=32.1 ; \mathrm{df}=4\right)$ \\
& 5 & 5 & 34 & \\
& 1 & 317 & 246 & 171 \\
& 2 & 149 & 30 & \\
& 3 & 17 & 14 & \\
\hline
\end{tabular}

Expressed ambiguous and unambiguous 10 base pair tags for two LongSAGE libraries were distinguished based on the number of 17 base pair sequences that give rise to the same tag. The tags in each of these two groups were binned according to the number of estimated mixture components. The Chi-square statistic was used to test the null hypothesis that these two groups are equivalent. 
Table 3: Top component memberships

\begin{tabular}{|c|c|c|}
\hline Dataset & & \\
\hline Component assignment & Freq. & p-value \\
\hline $\begin{array}{l}\text { BRAIN } \\
\text { astrocytoma }\left(N=14 n_{k=2}=4\right.\end{array}$ & & \\
\hline$-+----1--1 k=2$ & 14 & $4.3 \mathrm{E}-28$ \\
\hline---+++++++++++ & 12 & 2.7E-23 \\
\hline ependymoma $\left(N=10 n_{k=2}=\right.$ & & \\
\hline-+------ & 16 & $6.0 \mathrm{E}-14$ \\
\hline--+------ & 15 & $9.3 \mathrm{E}-13$ \\
\hline glioblastoma $\left(\mathrm{N}=7 \mathrm{n}_{\mathrm{k}=2}=60\right.$ & & \\
\hline---+--- & 48 & $3.2 \mathrm{E}-19$ \\
\hline-----+ & 42 & $5.2 \mathrm{E}-15$ \\
\hline medulloblastoma $\left(\mathrm{N}=18 \mathrm{n}_{\mathrm{k}}=\right.$ & & \\
\hline 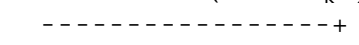 & 4 & $1.5 \mathrm{E}-9$ \\
\hline---------+----+ & 4 & $1.5 \mathrm{E}-9$ \\
\hline $\operatorname{normal}\left(N=9 n_{k=2}=588\right)$ & & \\
\hline-----+-- & 41 & $7.4 \mathrm{E}-37$ \\
\hline-----+-+ & 21 & $7.6 \mathrm{E}-14$ \\
\hline AML & & \\
\hline $\operatorname{inv}(16)\left(N=5 n_{k=2}=387\right)$ & & \\
\hline-++++ & 110 & $2.5 \mathrm{E}-39$ \\
\hline-+-++ & 36 & 0.028 \\
\hline$t(8 ; 2 \mathrm{I})\left(\mathrm{N}=5 \mathrm{n}_{\mathrm{k}=2}=387\right)$ & & \\
\hline$-++--\quad k=2 \quad k$ & 110 & $2.5 \mathrm{E}-39$ \\
\hline-++-+ & 36 & 0.028 \\
\hline$t(15 ; 17)\left(N=5 n_{k=2}=225\right)$ & & \\
\hline-++-- & 110 & $4.5 \mathrm{E}-67$ \\
\hline-++-+ & 36 & $1.0 \mathrm{E}-6$ \\
\hline $\mathrm{t}(9 ; 1 \mathrm{I})$ de novo $\left(\mathrm{N}=4 \mathrm{n}_{\mathrm{k}=2}=\right.$ & & \\
\hline-+-+ & 143 & $1.6 \mathrm{E}-16$ \\
\hline-+-- & 132 & $1.4 \mathrm{E}-12$ \\
\hline $\mathrm{t}(9 ; \mathrm{II})$ treated $\left(\mathrm{N}=3 \mathrm{n}_{\mathrm{k}=2}=\right.$ & & \\
\hline-++ & 216 & I.IE-I6 \\
\hline BREAST & & \\
\hline $\operatorname{normal}\left(\mathrm{N}=6 \mathrm{n}_{\mathrm{k}=2}=57 \mathrm{I}\right)$ & & \\
\hline---+-- & 82 & $3.6 \mathrm{E}-29$ \\
\hline-----+ & 65 & $4.0 \mathrm{E}-18$ \\
\hline $\operatorname{DCIS}\left(N=4 n_{k=2}=154\right)$ & & \\
\hline--++ & 97 & I.4E-43 \\
\hline-+++ & 41 & $4.5 \mathrm{E}-5$ \\
\hline invasive $\left(N=3 n_{k=2}=765\right)$ & & \\
\hline-+- & 337 & $4.6 \mathrm{E}-10$ \\
\hline SKIN & & \\
\hline $\operatorname{normal}\left(\mathrm{N}=4 \mathrm{n}_{\mathrm{k}=2}=500\right)$ & & \\
\hline---+ & 215 & I.8E-54 \\
\hline melanoma $\left(N=3 n_{k=2}=650\right)$ & & \\
\hline-+- & 405 & 2.|E-5| \\
\hline
\end{tabular}

For each set of biological replicates, the top one or two component states were selected from tags where the estimated number of components is 2. One component was represented with -, the other with + (i.e. -+- is equivalent to +-+). The significance was calculated using a zero-truncated Binomial test. The number of possible ways for the libraries to be assigned to the two components is $(2 \mathrm{~N} / 2)-\mathrm{I}$, where $\mathrm{N}$ is the number of libraries.

found highly significant in one test tend to be so in the other (Figure 2).

However, a number of observations are found significant using the overdispersed log-linear model and not the
Poisson mixture model, and vice versa. A closer look at the most extreme examples illustrates the superior performance of the mixture approach (Figure 3). In the first example, tag ACAACAAAGA seems clearly expressed in normal libraries, but is completely abolished in the ependymoma 


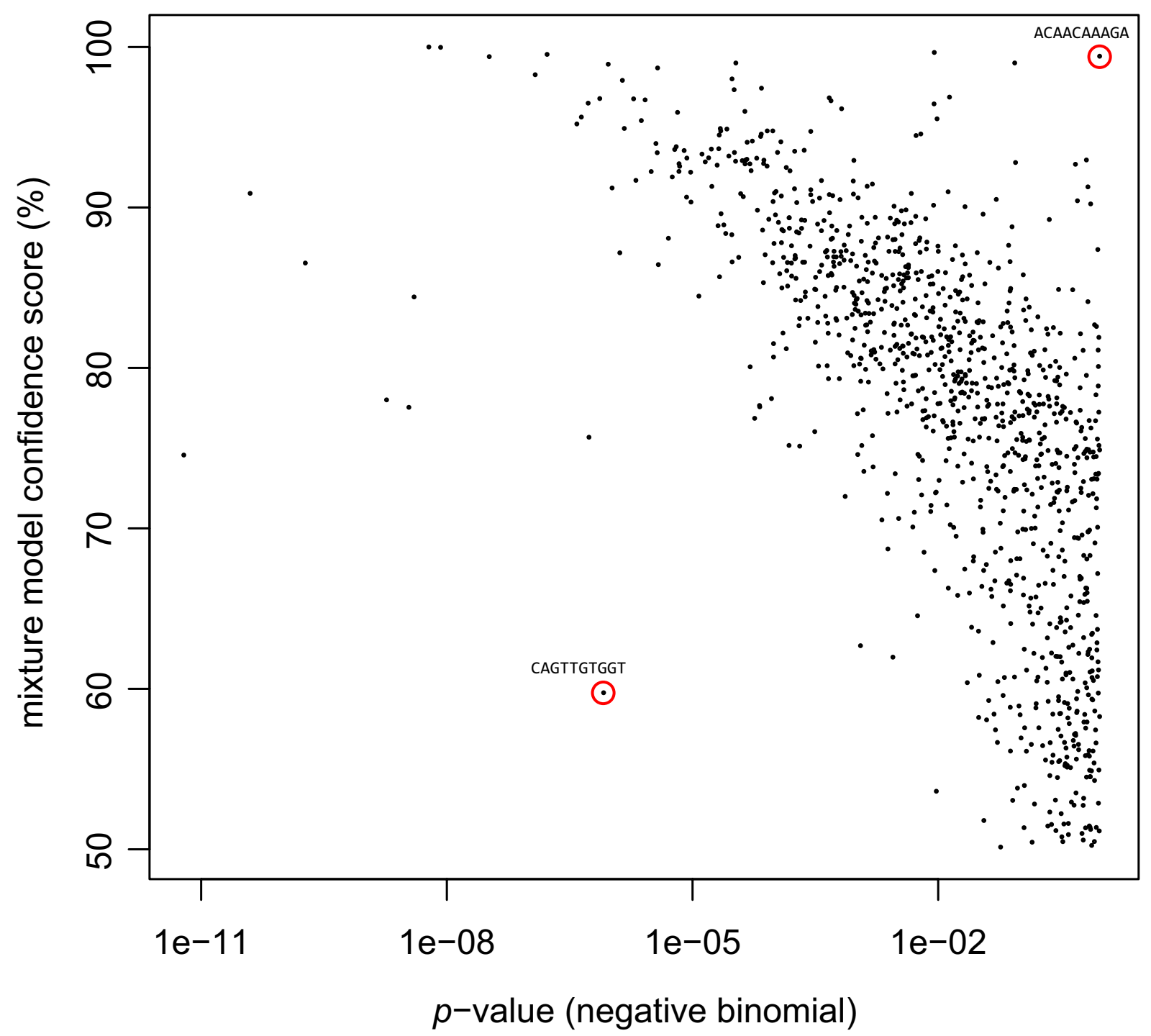

Figure 2

Comparison to significance scores for a test of differential expression calculated using a negative binomial model. Using the tag counts from 8 normal brain libraries versus 10 ependymoma libraries, differential expression between these two sample types was assessed using two methods. Plotted are the significance scores calculated for a negative binomial model versus a Poisson mixture model. The negative binomial (x-axis) is a $p$-value, so smaller values are more significant. The Poisson mixture (y-axis) is a confidence score, so larger values are more significant. Circled are two examples of SAGE tags where one model shows significance while the other does not.

libraries. However, according to the overdispersed model, the observation is not at all significant $(p=0.9998)$. The mixture model, however, produces a confidence score of
$99.42 \%$, which suggests this tag is highly informative with respect to sample type. This example demonstrates the difficulty that the log-linear model has with fitting groups 


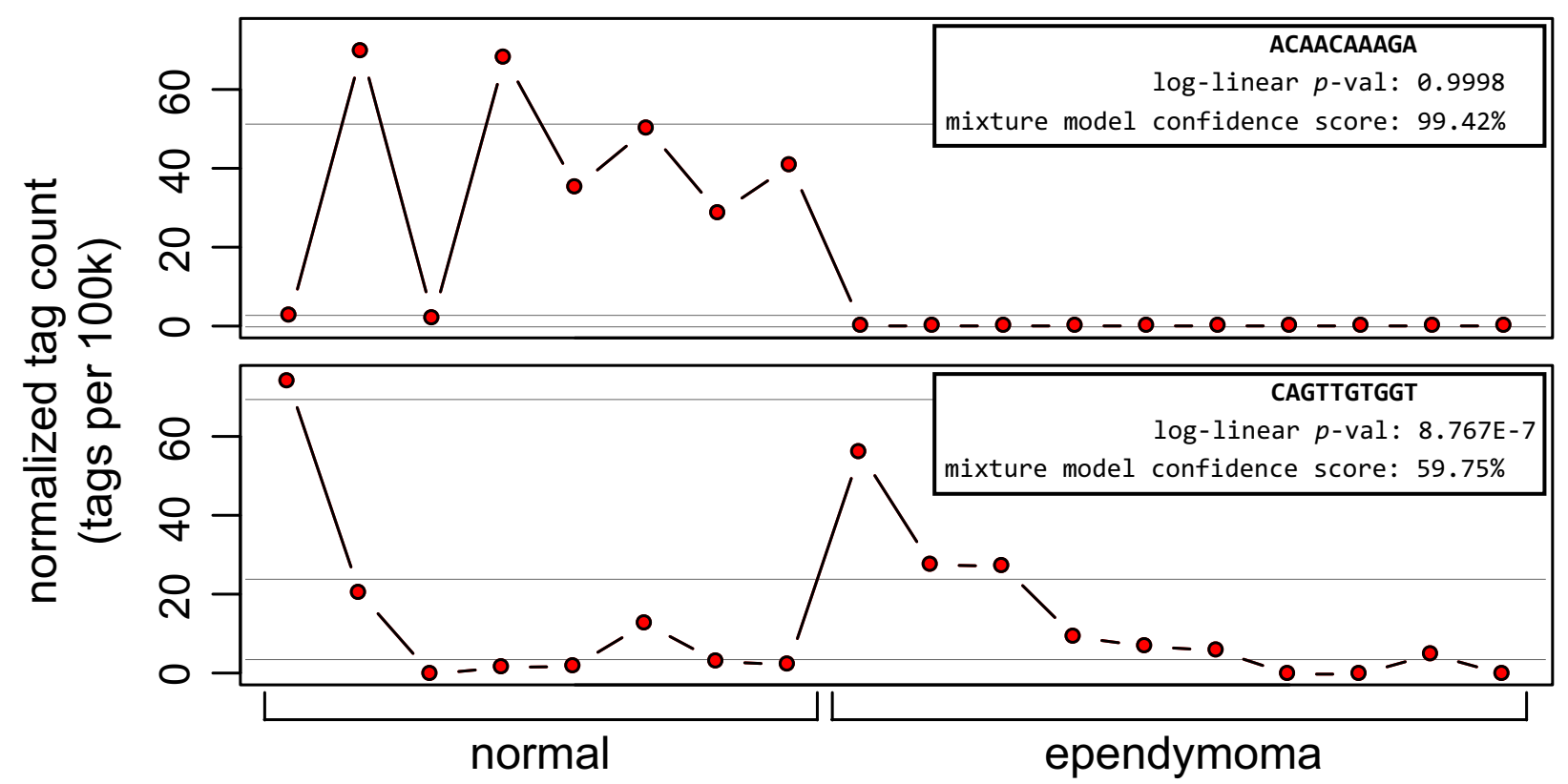

\section{Figure 3}

Counts for two tags assessed using a negative binomial model and the Poisson mixture model where one model shows significance and the other does not. The figure is divided to show separate plots of the expression level of two tags observed in 8 normal brain libraries and 10 ependymoma libraries. The $x$-axis is the normalized expression (count/ library size*100,000) and the $y$-axis is divided into the two sample types. In the top plot, the negative binomial model is not significant and the Poisson mixture is significant; in the bottom plot, the situation is reversed. Light gray guide lines denote the expected expression level of the Poisson components.

where tag counts are zero, a problem that is even more pronounced when using a logistic regression model (for a more thorough discussion of this problem see [6]).

In the second example, tag CAGTTGTGGT clearly has increased expression in some libraries from both the normal and ependymoma groups. However, according to the overdispersed model, the observation is highly significant $(p=8.8 \mathrm{E}-7)$. The mixture model, however, produces a confidence score of $59.8 \%$ which is only nominally better than chance. This example demonstrates how the log-linear model seems to downweight the occasional extreme observation in one group, even if it is in agreement with observations in the other group. This can result in candidate lists based on the log-linear significance containing tags that have extreme observations that occur at a higher rate in one group over another, which are typically of little interest.

Similar results were obtained when comparing to the Bayes error rate described in [7]. Again, a moderate correlation is seen and tags found highly significant in one test tend to be so in the other (Figure 4). Overall, the Bayes error rate is in better agreement with the mixture model confidence score and appears to be more robust in assessing tags with zero counts in one group. However, the assumption of a hierarchical model (in this case, a betabinomial) used to calculate the Bayes error rate versus a Poisson mixture model results in differences between the two methods. Two examples, analogous to those described above, are highlighted (Figure 5). In both cases, the Poisson mixture model appears to give confidence values that are in better agreement with the observations.

\section{Discussion}

The exploration of statistical approaches to SAGE analysis is important since the number of studies using the technology has resulted in a continuing rise in the amount of available data. The notion of sampling variability being the predominant source of "within"-library variability and distinct components being the predominant source of "between"-library variability is reassuring for investigators who choose the SAGE technique to obtain a comprehensive profile of gene expression in a limited number of samples. Nevertheless, there is certainly a contribution by a latent biological variability as evidenced by the increased performance of the negative binomial as the number of libraries increases. However, this work demon- 


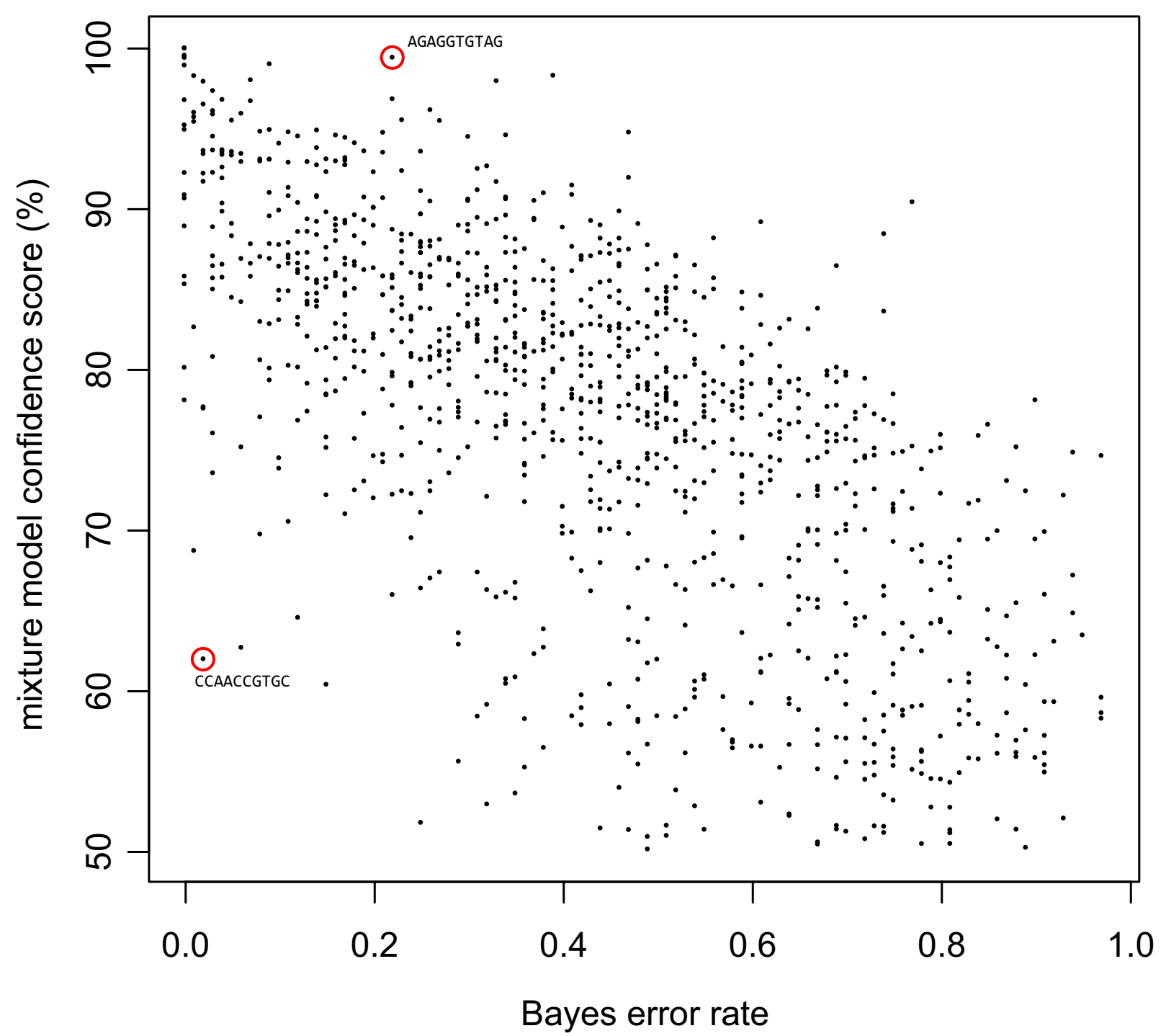

\section{Figure 4}

Comparison to Bayes error rate for a test of differential expression calculated using a beta binomial model. Using the tag counts from 8 normal brain libraries versus 10 ependymoma libraries, differential expression between these two sample types was assessed using two methods. Plotted are the Bayes error rate described in [7] versus a Poisson mixture model confidence score. For the Bayes error rate ( $x$-axis) smaller values are more significant. The Poisson mixture ( $y$-axis) is a confidence score, so larger values are more significant. Circled are two examples of SAGE tags where one model shows significance while the other does not. 


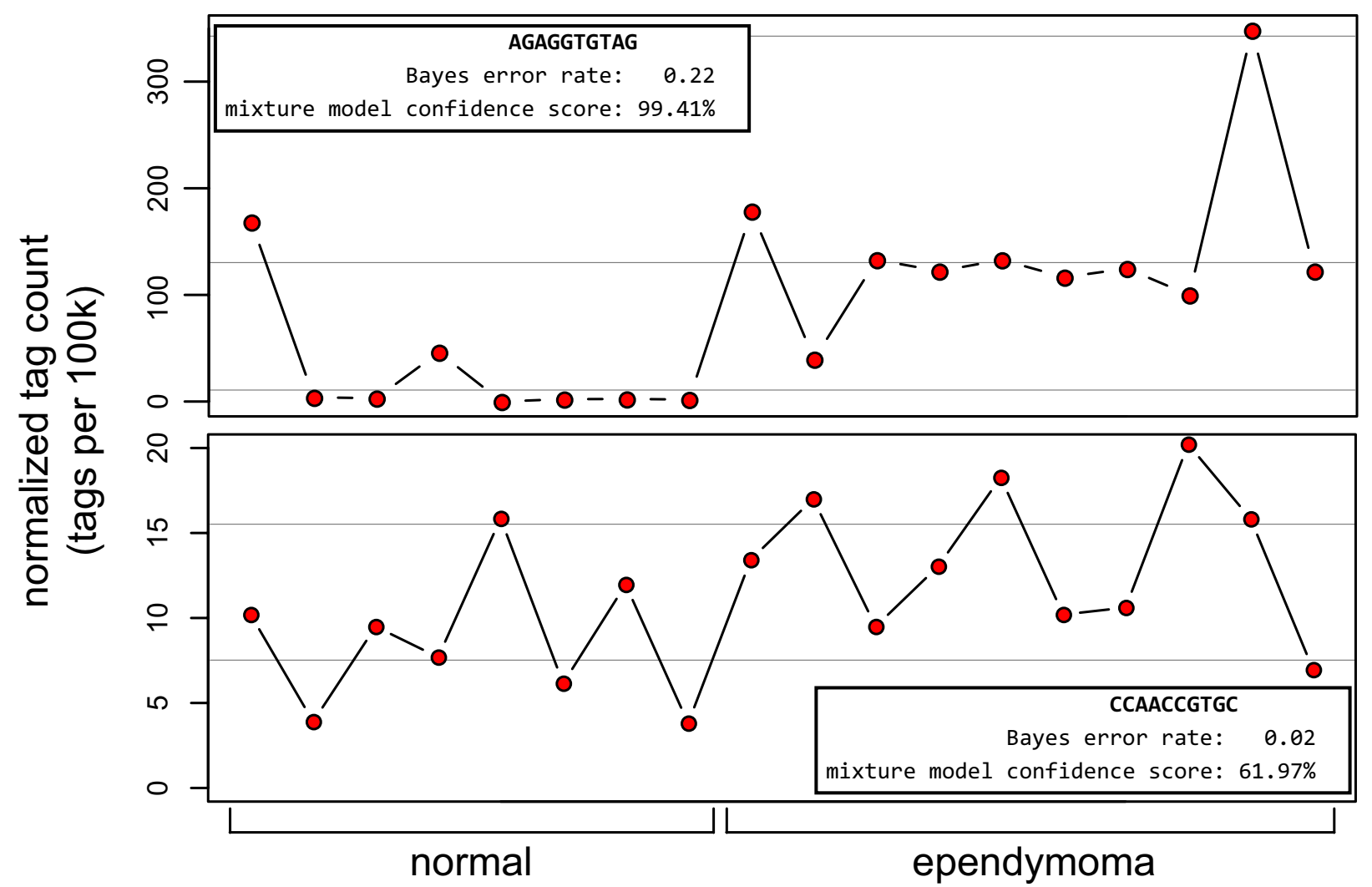

\section{Figure 5}

Counts for two tags assessed using a Bayes error rate and the Poisson mixture model where one model shows significance and the other does not. The figure is divided to show separate plots of the expression level of two tags observed in 8 normal brain libraries and 10 ependymoma libraries. The $x$-axis is the normalized expression (count/library size*100,000) and the $y$-axis is divided into the two sample types. In the top plot, the Bayes error rate is not significant and the Poisson mixture is significant; in the bottom plot, the situation is reversed. Light gray guide lines denote the expected expression level of the Poisson components.

strates that a simple overdispersed model may overstate this effect, and that certainly there is a clustering of expression into distinct components, which are then sampled. This is consistent with the view of gene transcription for any one locus consisting of (possibly several) inactivated or activated state(s). The same idea holds for some known mechanisms of genetic disease, such as loss of heterozygosity (LOH) or amplification of a particular locus (e.g. cancer).

For this reason, it is recommended that investigators try the mixture model approach in comparisons of groups of biological replicates. Failing this, some of the difficulties that can be encountered with the negative binomial model can be lessened by: a) setting a tolerance for how much overdispersion $(\varphi)$ is acceptable in a final list of candidate tags, although such a cutoff would be somewhat arbritrary; and b) add a small value to the tag count to avoid the problems the model has with groups consisting of many zero counts. One strategy is to assume equal odds that the next tag drawn is the one of interest by adding 1 to the count, and 2 to the library size (i.e. (count+1)/ $($ size+2)) (K. Baggerly, personal communication).

In the future, it may be worthwhile to combine both approaches by defining a negative binomial mixture model. However, at this point, such an approach is unlikely to provide significant improvement given the small number of libraries in a typical set of available biological replicates. In addition, applying the concept of "information sharing" between tags may provide estimates of statistically informative variables that apply library-wide, and could be utilized to improve the power of the method described in this paper $[11,12]$. 


\section{Conclusion}

The Poisson mixture model appears to be a rational means to represent SAGE data that are biological replicates and as a basis to assign significance when comparing multiple groups of such replicates. The use of a mixture model can improve the process of selecting differentially expressed genes, and provide a foundation for $a b$ initio identification of co-expressed genes and/or biologicallyrelevant sample subsets.

\section{Methods}

\section{Test datasets}

Test datasets were obtained from the Gene Expression Omnibus (GEO) [13] and reflect a range of cancer studies, including malignancies of the skin [14-16], breast [1719], blood [20], and brain [21]. The full description, accession, and size for each library were recorded [see Additional file 2]. In the case of breast and skin data, libraries from a combination of studies were used. Datasets were filtered to remove tags expressed at a mean less than 100 tags per million.

\section{Model fitting}

The open-source statistical software package $\mathrm{R}$ was used to perform all calculations in this paper [22]. R code is included with the explanation for each model. For each of the models, let $Y_{i}$ be the observed tag count in library $i, n_{i}$ be the total number of tags in library $i$, and $N$ be the total number of libraries. Also, let $x_{\mathrm{i}}$ be the vector of explanatory variables (e.g. normal $=0$ and cancer $=1$ ) associated with the library $i$, and $\beta$ be the vector of coefficients.

\section{Log-linear (Poisson) regression model}

The log-linear model assumes that the observed tag counts are distributed as

$\mathrm{Y}_{\mathrm{i}} \sim \operatorname{Poisson}\left(\mu_{\mathrm{i}}\right)$

$\mu_{\mathrm{i}}=\mathrm{n}_{\mathrm{i}} \mathrm{p}_{\mathrm{i}}$

where $\mathrm{p}_{\mathrm{i}}$ is the actual expression in terms of the proportion of all expressed tags.

Here, the unconditional mean and variance are $\mathrm{E}\left(\mathrm{Y}_{\mathrm{i}}\right)=$ $\operatorname{Var}\left(\mathrm{Y}_{\mathrm{i}}\right)=\mu_{\mathrm{i}}$. Using the log link function, which linearizes the relationship between the dependent variables and the predictor(s), we obtain the linear equation

$\log \left(\mathrm{Y}_{\mathrm{i}}\right)=\log \left(\mathrm{n}_{\mathrm{i}}\right)+x_{\mathrm{i}} \beta$

$\mathrm{p}_{\mathrm{i}}=\exp \left(x_{\mathrm{i}} \beta\right)$

Using iteratively reweighted least-squares (IRLS), the value(s) for the coefficient(s) $\beta$ are estimated. The stats library included with R can fit a log-linear model using the following code:

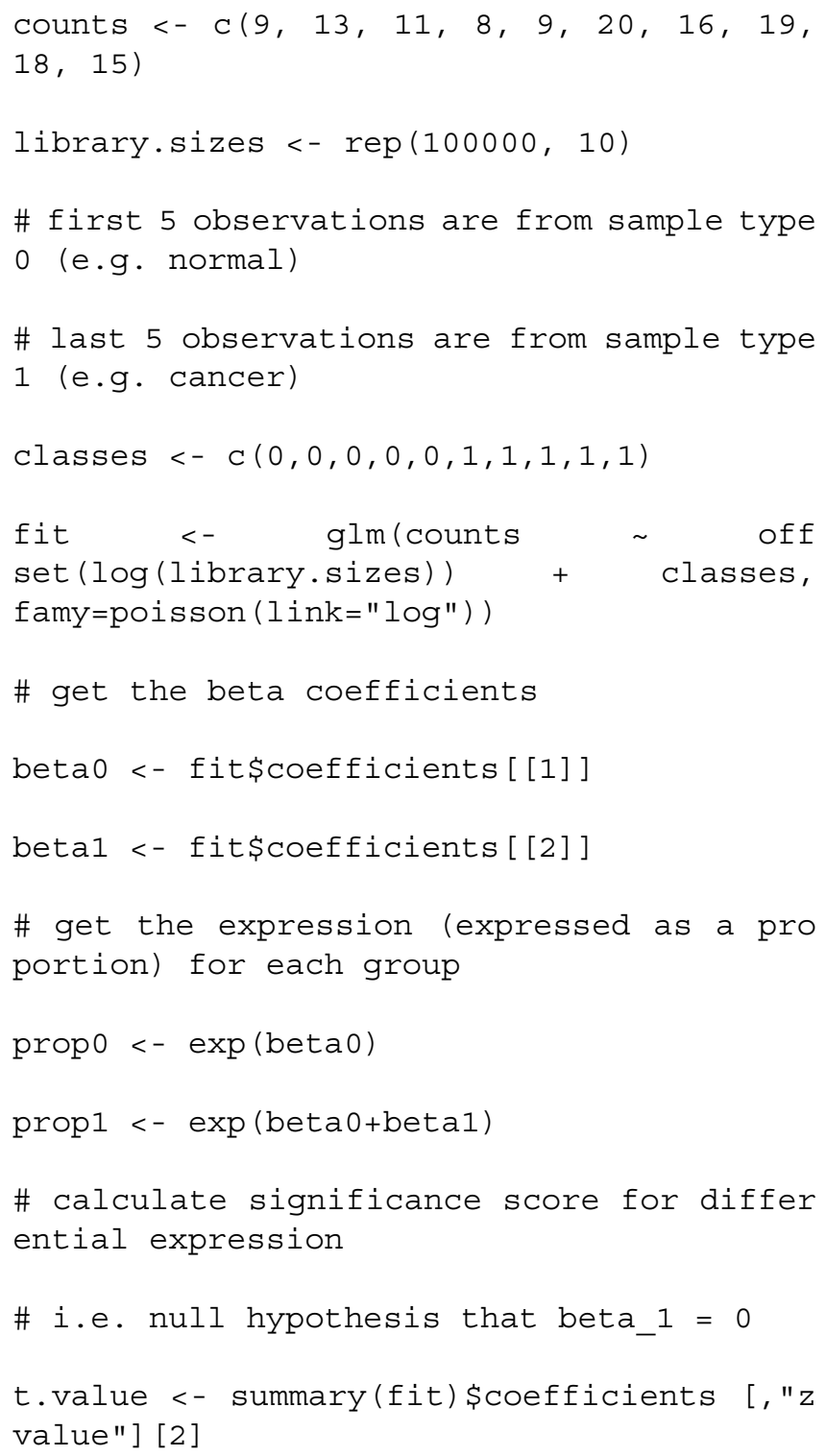

\section{Overdispersed log-linear regression model}

In contrast to the canonical log-linear model, we assume the actual expression is distributed as

$\theta_{\mathrm{i}} \sim \operatorname{Gamma}\left(\mu_{\mathrm{i}}, 1 / \varphi\right)$

$\mu_{\mathrm{i}}=\mathrm{n}_{\mathrm{i}} \mathrm{p}_{\mathrm{i}}$

where, as above, $\mathrm{p}_{\mathrm{i}}$ is the actual expression in terms of the proportion of all expressed tags. Here, the unconditional mean and variance are $\mathrm{E}\left(\theta_{\mathrm{i}}\right)=\mu_{\mathrm{i}}$ and $\operatorname{Var}\left(\theta_{\mathrm{i}}\right)=\mu_{\mathrm{i}}^{2} \varphi$. Since 
we are now sampling from this latent Gamma distribution, the observed tag counts are conditional on this underlying expression and are distributed as

$\mathrm{Y}_{\mathrm{i}} \mid \mathrm{p}_{\mathrm{i}}, \varphi \sim \operatorname{Poisson}\left(\theta_{\mathrm{i}}\right)$

Now, the unconditional mean and variance are $\mathrm{E}\left(\mathrm{Y}_{\mathrm{i}}\right)=\mu_{\mathrm{i}}$ and $\operatorname{Var}\left(\mathrm{Y}_{\mathrm{i}}\right)=\mu_{\mathrm{i}}\left(1+\mu_{\mathrm{i}} \varphi\right)$.

As above, using the log link function we obtain the linear equation

$\log \left(\mathrm{Y}_{\mathrm{i}}\right)=\log \left(\mathrm{n}_{\mathrm{i}}\right)+x_{\mathrm{i}} \beta$

$\mathrm{p}_{\mathrm{i}}=\exp \left(x_{\mathrm{i}} \beta\right)$

Here, a maximum likelihood estimate of the values for the coefficient(s) $\beta$ and the overdispersion parameter $(\varphi)$ can be performed. The MASS library [23] for R can fit an overdispersed log-linear model using the following code:

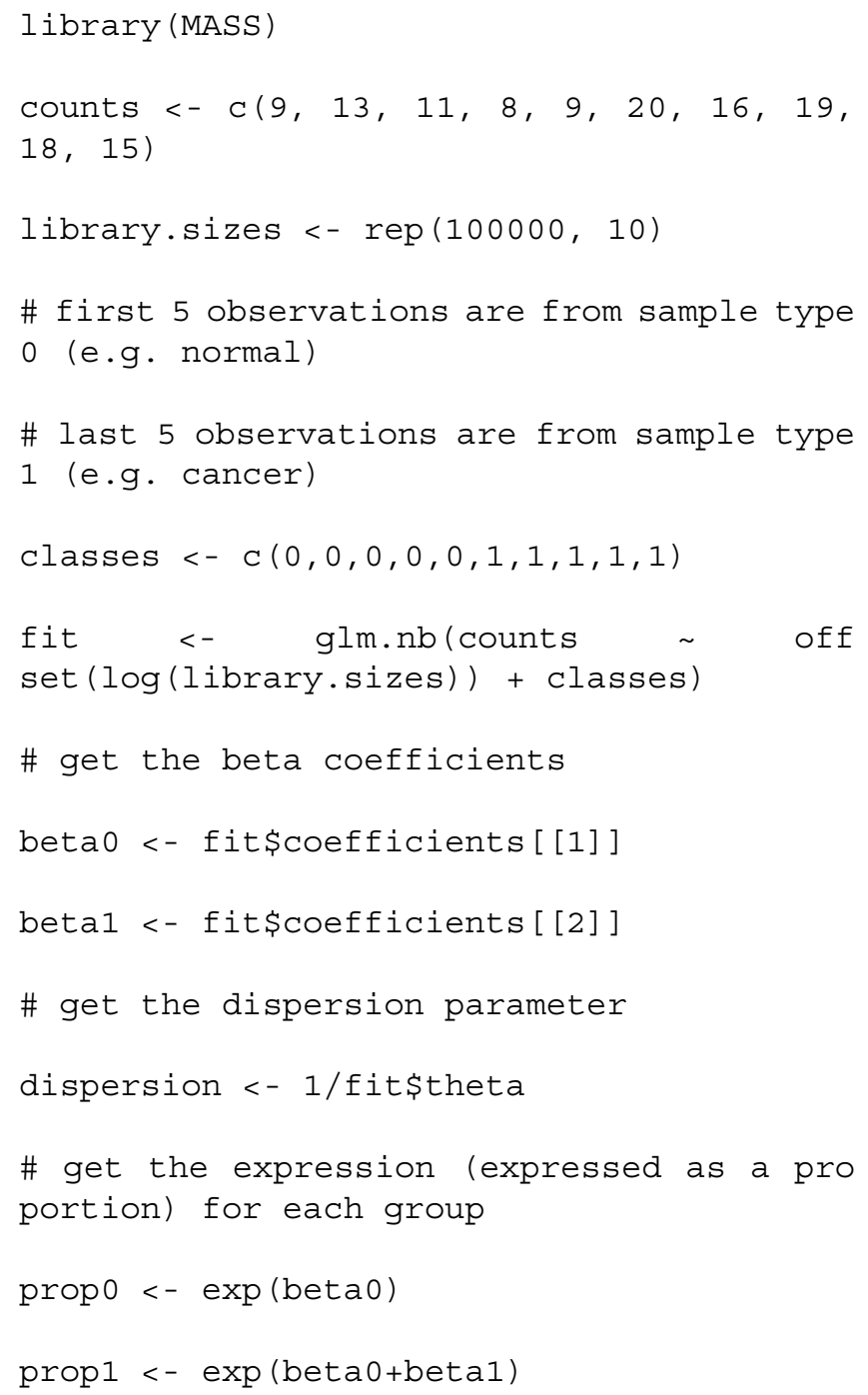

\# calculate significance score for differ ential expression

\# i.e. null hypothesis that beta_1 $=0$

t. value <- summary(fit)\$coefficients $[, " z$ value"] [2]

p.value <- 2 * pt (-abs (t.value), fit\$df.residual)

A more complete discussion of this model and its application to SAGE, including significance testing, is described in [6].

\section{Poisson mixture model}

Like the canonical log-linear regression model, we assume the observed tag counts are Poisson distributed. However, the counts are conditional on the choice of a Poisson-distributed component, such that

$\mathrm{Y}_{\mathrm{i}} \mid \mathrm{k} \sim \operatorname{Poisson}\left(\mu_{\mathrm{ik}}\right)$

$\mu_{\mathrm{ik}}=\mathrm{n}_{\mathrm{i}} \mathrm{p}_{\mathrm{ik}}$

where the component $\mathrm{k}=1,2, \ldots, \mathrm{K}$ and $\mathrm{p}_{\mathrm{ik}}$ is the actual expression for component $\mathrm{k}$ in terms of the proportion of all expressed tags. The posterior probability that an observed tag count belongs to a component $\mathrm{k}$ is given by

$$
P\left(k \mid Y_{i}, \psi\right)=\frac{\pi_{k} f\left(Y_{i} \mid \mu_{i k}\right)}{\sum_{j}^{K} f\left(Y_{i} \mid \mu_{i j}\right)}
$$

where $\psi$ is the parameter vector containing the component means $\left(\theta_{1}, \ldots, \theta_{\mathrm{K}}\right)$ and mixing coefficients $\left(\pi_{1}, \ldots \pi_{\mathrm{K}-1}\right)$. $\mathrm{f}($.) is the Poisson probability density function. To fit the model, one must estimate the values in $\psi$. This can be done by maximum likelihood estimation (MLE) using the EM algorithm [24]. The flexmix library for R uses the EM algorithm to fit a variety of finite mixture models [25]. In the case of SAGE data, the following code can be used:

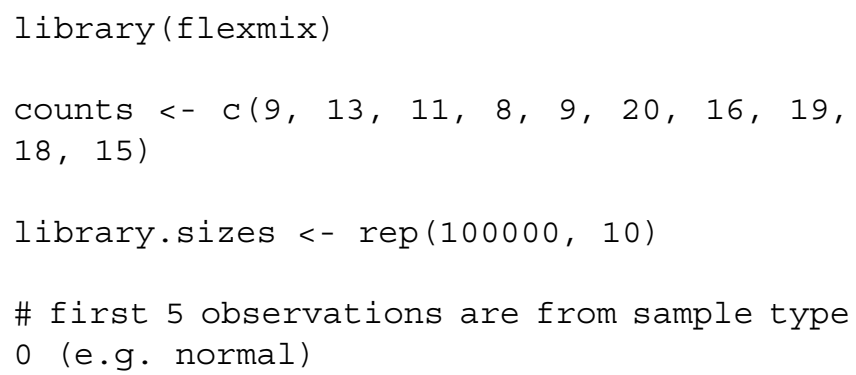


\# last 5 observations are from sample type 1 (e.g. cancer)

classes <- C $(0,0,0,0,0,1,1,1,1,1)$

\# set fitting control parameters to set tings that work

\# well for SAGE

custom. FLXcontrol <- list (iter. $\max =200$,

minprior=0,

tolerance $=1 \mathrm{E}-6$,

verbose=0,

classify="hard",

nrep=1)

custom.FLXcontrol <- as(custom.FLXcon

trol, "FLXcontrol")

\# specify the maximum number of model com ponents

$\operatorname{maxk}<-5$

fits $<-$ list ()

aic.fits <- rep (NA, maxk)

\# increase number of components until AIC decreases

for $(\mathrm{k}$ in $1: \operatorname{maxk}) \quad\{$

\# make an initial "good" guess of class membership

\# using k-means - helps avoid falling into a local

\# likelihood maximum

cm $<-\operatorname{rep}(1$, length (counts))

if $(\mathrm{k}>1) \mathrm{cm}<-\operatorname{kmeans}(($ count $\mathrm{s}+1) /$ $($ sizes+2),

centers=k) \$cluster

fit $<-$ try $($ flexmix (counts $\sim 1$, $\mathrm{k}=\mathrm{k}$ ，

model=FLXglm (family="poisson", offset $=\log ($ sizes $))$,

control=custom. FLXcontrol,

cluster $=\mathrm{cm})$, silent $=$ TRUE )

if("try-error" \%in\% class(fit)) break

\# stop if there were less components found then

\# specified

if (max (cluster(fit)) > k) break

fits $[\mathrm{k}]]<-$ fit

aic.fits [k] <-AIC(fits [k]])

if $(\mathrm{k}==1)$ next

if(aic.fits [k] >= aic.fits [k-1]) break

\}

\# what number of components minimized AIC?

k.optimal <- which(aic.fits == min(aic.fits, na.rm=TRUE)) [1]

fit $<-$ fits [k.optimal]]

\# get the theta parameters

thetas <- array (dim=k.optimal)

for (i in 1:k.optimal) thetas [i] <- param eters (fit, component=i) \$coef

\# get the pi parameters

pis <- attributes (fit) \$prior

\# what is the confidence score that the fitted components

\# differentiate between groups?

confidence <- pmm. confidence(fit, classes, use. scaled=FALSE) 
The confidence score, ranging from $50-100 \%$, is explained in the Results section and code for performing the calculation is available [see Additional file 3].

\section{Authors' contributions}

SDZ conceived of, developed, and tested the presented research.

\section{Additional material}

\section{Additional file 1 \\ Derivation of confidence score. A derivation of a confidence score for dif- ferential expression based on a Poisson mixture model fit. \\ Click here for file \\ [http://www.biomedcentral.com/content/supplementary/1471- 2105-8-282-S1.pdf] \\ Additional file 2 \\ Supplementary SAGE library information. An Excel spreadsheet contain- ing accessions, sizes, and descriptions of the libraries included in this study. \\ Click here for file \\ [http://www.biomedcentral.com/content/supplementary/1471- 2105-8-282-S2.xls] \\ Additional file 3 \\ Confidence score $R$ function. $R$ source code for a function to calculate the differential expression confidence score based on a Poisson mixture model fit. \\ Click here for file \\ [http://www.biomedcentral.com/content/supplementary/1471- 2105-8-282-S3.R]}

\section{Acknowledgements}

SDZ is supported by the Canadian Institutes for Health Research (CIHR) and the Michael Smith Foundation for Health Research (MSFHR). Computationally intensive portions of this work were made possible by the WestGrid computing resources http://www.westgrid.ca, which are funded in part by the Canada Foundation for Innovation, Alberta Innovation and Science, $B C$ Advanced Education and the participating research institutions. WestGrid equipment is provided by IBM, Hewlett Packard and SGI. The author thanks Dr. Greg Vatcher for critical reading of the manuscript, and Drs. Raymond Ng and Victor Ling for helpful advice.

\section{References}

I. Velculescu VE, Zhang L, Vogelstein B, Kinzler KW: Serial analysis of gene expression. Science 1995, 270(5235):484-487.

2. Saha S, Sparks AB, Rago C, Akmaev V, Wang CJ, Vogelstein B, Kinzler $\mathrm{KW}$, Velculescu VE: Using the transcriptome to annotate the genome. Nature biotechnology 2002, 20(5):508-5I2.

3. Matsumura H, Reich S, Ito A, Saitoh H, Kamoun S, Winter P, Kahl G, Reuter M, Kruger DH, Terauchi R: Gene expression analysis of plant host-pathogen interactions by SuperSAGE. Proceedings of the National Academy of Sciences of the United States of America 2003, I00(26): I57|8-|5723.

4. Baggerly KA, Deng L, Morris JS, Aldaz CM: Differential expression in SAGE: accounting for normal between-library variation. Bioinformatics 2003, 19(12): |477-1483.

5. Baggerly KA, Deng L, Morris JS, Aldaz CM: Overdispersed logistic regression for SAGE: modelling multiple groups and covariates. BMC Bioinformatics 2004, 5: 144.
6. Lu J, Tomfohr JK, Kepler TB: Identifying differential expression in multiple SAGE libraries: an overdispersed log-linear model approach. BMC Bioinformatics 2005, 6:165.

7. Vencio RZ, Brentani H, Patrao DF, Pereira CA: Bayesian model accounting for within-class biological variability in Serial Analysis of Gene Expression (SAGE). BMC Bioinformatics 2004, 5:119.

8. McLachlan GJ, Peel D: Finite mixture models. New York: Wiley; 2000.

9. Akaike $\mathrm{H}$ : A new look at the statistical model identification. IEEE Transactions on Automatic Control 1974, I 9(6):716-723.

10. Schwarz G: Estimating the dimension of a model. Annals of Statistics 1978, 6(2):46I-464.

II. Kuznetsov VA, Knott GD, Bonner RF: General statistics of stochastic process of gene expression in eukaryotic cells. Genetics 2002, I 6 I(3): I32I-I332.

12. Thygesen HH, Zwinderman AH: Modeling Sage data with a truncated gamma-Poisson model. BMC Bioinformatics 2006, 7:157.

13. Edgar R, Domrachev M, Lash AE: Gene Expression Omnibus: NCBI gene expression and hybridization array data repository. Nucleic acids research 2002, 30(I):207-210.

14. Cornelissen M, van der Kuyl AC, van den Burg R, Zorgdrager F, van Noesel Cl, Goudsmit ]: Gene expression profile of AIDS-related Kaposi's sarcoma. BMC Cancer 2003, 3:7.

15. van Ruissen F, Jansen BJ, de Jongh GJ, Zeeuwen PL, Schalkwijk J: A partial transcriptome of human epidermis. Genomics 2002, 79(5):67I-678.

16. Weeraratna AT, Becker D, Carr KM, Duray PH, Rosenblatt KP, Yang S, Chen Y, Bittner M, Strausberg RL, Riggins GJ, et al.: Generation and analysis of melanoma SAGE libraries: SAGE advice on the melanoma transcriptome. Oncogene 2004, 23( I 2):2264-2274.

17. Porter D, Lahti-Domenici J, Keshaviah A, Bae YK, Argani P, Marks J, Richardson A, Cooper A, Strausberg R, Riggins G], et al:: Molecular markers in ductal carcinoma in situ of the breast. Mol Cancer Res 2003, I(5):362-375.

18. Porter D, Weremowicz S, Chin K, Seth P, Keshaviah A, Lahti-Domenici J, Bae YK, Monitto CL, Merlos-Suarez A, Chan J, et al.: A neural survival factor is a candidate oncogene in breast cancer. Proceedings of the National Academy of Sciences of the United States of America 2003, I00( I 9): 10931-10936.

19. Porter DA, Krop IE, Nasser S, Sgroi D, Kaelin CM, Marks JR, Riggins G, Polyak K: A SAGE (serial analysis of gene expression) view of breast tumor progression. Cancer Res 200I, 6 I ( I 5):5697-5702.

20. Lee S, Chen J, Zhou G, Shi RZ, Bouffard GG, Kocherginsky M, Ge X, Sun M, Jayathilaka N, Kim YC, et al.: Gene expression profiles in acute myeloid leukemia with common translocations using SAGE. Proceedings of the National Academy of Sciences of the United States of America 2006, I 03(4): 1030-1035.

21. Boon K, Edwards JB, Eberhart CG, Riggins GJ: Identification of astrocytoma associated genes including cell surface markers. BMC Cancer 2004, 4:39.

22. R Development Core Team: A Language and Environment for Statistical Computing. R Foundation for Statistical Computing 2006.

23. Venables WN, Ripley BD: Modern Applied Statistics with S. Fourth edition. New York: Springer; 2002.

24. Dempster A, Laird N, Rubin D: Maximum Likelihood from Incomplete Data via the EM-Algorithm. Journal of the Royal Statistical Society Series B (Methodological) 1977, 39(I): I-38.

25. Leisch F: FlexMix: A general framework for finite mixture models and latent class regression in R. Journal of Statistical Software 2004, I I : 